\title{
Awareness of Vitamin D Deficiency among Females Attending the OPD, AIIMS,Rishikesh.
}

\author{
Mrs. Vasantha Kalyani ${ }^{1}$, Mrs. Shaina Sharma ${ }^{2}$ \\ ${ }^{I}$ Assistant Professor, College of Nursing, AIIMS, Rishikesh. \\ ${ }^{2}$ Nursing Tutor, College of Nursing, AIIMS, Rishikesh.
}

\begin{abstract}
Introduction:Vitamin D deficiency is a globally occurring problem nowadays. Most of the Indians are suffering from this deficiency which may be due to the inadequate exposure to the sunlight, inappropriate infrastructure, cultural or religious practices, inadequate consumption of the dairy products fortified with Vitamin D. This study was conducted to know the awareness of the females attending the OPDs at AIIMS, Rishikesh.

Materials and Methods:A structured interview schedule consisting of 10 questions on sociodemographic profile and 13 questions related to awareness about vitamin D was administered to the target population of 100 females.

Results:Majority of the females (39\%) are from the age group 20-30 years. Most of the females (66\%) reside in the urban area. $57 \%$ of the females have the wheatish complexion followed by fair (31\%) and dark complexion $(12 \%)$.Most of the females (86\%) never got themselves tested for vitamin D levels in their body. $80 \%$ of them are suffering with leg pain/ back pain or bone pain. Majority of the females (60\%) are not aware that vitamin D deficiency can occur at any age. Most of them (56\%) are also not aware that vitamin D helps in curing the calcium deficiency. Conclusion: Vitamin D deficiency is prevalent among the various age groups in our country due to various factors. So, Awareness programmes have to be conducted to make the general public including the females aware of the vitamin D, its deficiency, causative factors and the preventive measures.
\end{abstract}

\section{Introduction}

Vitamin D is vital for the bone health as it helps in proper utilization of calcium in our diet. It is produced due to the exposure of skin to the sun. It also occurs naturally in few of the food products like fortified dairy and grains, fish and fish liver oils. If the body is not getting the sufficient vitamin $\mathrm{D}$, it may lead to the vitamin D deficiency. This vitamin D deficiency is responsible for causing Rickets in children and Osteomalacia in adults. It plays an important role in the development of the cardiovascular disorders, osteoporosis, cancer, diabetes and infections ${ }^{1}$.

Vitamin D deficiency is the growing problem of today's world. The Indians suffer from the vitamin D deficiency as most of the students, professionals and the housewives don not come in contact with the sunlight ${ }^{2}$.

The prevalence of vitamin D deficiency in India is $70-100 \%$ in the general population. The reason behind this is the dairy food consumed in our country is not fortified with vitamin $\mathrm{D}$ and the cultural \& religious practices do not facilitate the exposure to the adequate sunlight ${ }^{1}$. Vitamin D deficiency is a big concern nowadays which has to be given attention so that essential steps can be taken towards its prevention.

\section{Need of the study}

Females are at high risk of calcium deficiency due to the hormonal changes in their body from time to time. If the females are deficient in vitamin D then the calcium deficiency may further worsens. Adequate amount of vitamin D in the body is essential for proper mineralization of the bones. Vitamin D deficiency can also cause the systemic diseases. It is essential to know about the understanding of the females about the vitamin $\mathrm{D}$ and its deficiency so that further steps can be taken.

Floor Christie and Linda Mason conducted a study to assess the knowledge, attitude and practice regarding vitamin D deficiency among female students in Saudi Arabia and found that the participants of the study were having limited knowledge about the vitamin D and its deficiency. The reasons behind this deficiency are limited exposure to sun, covering the body due to cultural practices and type of infrastructure limiting the sun exposure ${ }^{3}$.

There can be many reasons behind the vitamin D deficiency differing from culture to culture or country to country. So it is vital to know the knowledge or awareness of the females regarding the same. This study was undertaken to assess the awareness of the females regarding the vitamin D and its deficiency, who are attending the OPD at AIIMS, Rishikesh. 


\section{Problem statement}

A study to assess the awareness of Vitamin D deficiency among females attending the OPD, AIIMS, Rishikesh. Objective

To assess the awareness of Vitamin D deficiency among females

\section{Methodology}

Research design: An exploratory survey design

Population: Females

Target population: Females attending the OPD at AIIMS, Rishikesh.

Sampling technique: Convenience sampling

Sample size: 100

Data collection tool: Structured interview schedule on Awareness of Vitamin D deficiency consisting of 10 questions in demographic profile and 13 questions related to awareness regarding vitamin D deficiency.

Data analysis: Descriptive statistics

\section{Major findings of the study}

Table No. 1: Distribution of sample according to their Sociodemographic profile

$$
\mathrm{N}=100
$$

\begin{tabular}{|c|c|c|}
\hline 1. & Age & Frequency \& Percentage \\
\hline & a) 20-30 years & 39 \\
\hline & 31-40years & 18 \\
\hline & 41-50years & 27 \\
\hline & $>50$ years & 16 \\
\hline \multirow[t]{3}{*}{2.} & Residence & \\
\hline & a) Urban & 34 \\
\hline & b) Rural & 66 \\
\hline \multirow[t]{9}{*}{3.} & Occupation & \\
\hline & a) Housewife & 75 \\
\hline & b) Housekeeping staff & 03 \\
\hline & c) Accountant & 01 \\
\hline & d) Teacher & 03 \\
\hline & e) $\quad$ Student & 12 \\
\hline & f) Social worker & 02 \\
\hline & g) $\quad$ Laborer & 02 \\
\hline & h) $\quad$ Farmer & 02 \\
\hline \multirow[t]{3}{*}{4.} & Menstrual history & \\
\hline & Menstruating & 74 \\
\hline & Menopause & 26 \\
\hline \multirow[t]{4}{*}{5.} & Skin color & \\
\hline & a) Dark & 12 \\
\hline & b) Wheatish & 57 \\
\hline & c) Fair & 31 \\
\hline
\end{tabular}

Table 1 show that majority of the females (39\%) are from the age group 20-30years. Most of the females $(66 \%)$ reside in the urban area. Majority $(75 \%)$ of them are housewives. $74 \%$ of the females are menstruating while $26 \%$ have undergone menopause. $57 \%$ of the females have the wheatish complexion followed by fair $(31 \%)$ and dark complexion $(12 \%)$.

Others factors considered during the interview were as follows:--

Most of the females (98\%) do not smoke. Only 19\% of the total females use sunscreen. $79 \%$ of the women said they apply the sunscreen while going outside and $21 \%$ said they apply it always either while going out or staying at home. $42 \%$ of the females replied that they always cover their exposed skin while going outside. Majority of the females (79\%) did not have any history of metabolic disease while $21 \%$ said they have the history of metabolic disease.

Only $37 \%$ of the females are aware of the vitamin available for free. $20 \%$ of them said that they are taking some kind of diet supplements like iron, folic acid, calcium, vitamin D. Total $8 \%$ of the females are taking calcium supplements and $4 \%$ are taking the vitamin D supplements. When asked about the time spent in the sunlight daily $91 \%$ of the females said they spend some time of the day under the sun regularly. Out of these females (91\%), 39\% (36)spend less than 1 hour under the sunlight daily followed by 37\% (34) females who spend 1-2 hours in sunlight, $12 \%$ (11) 2-3hours and 10.9\% (10) more than 3 hours per day. Most of them (66\%) take the milk but only $50 \%$ of these females take milk daily. Most of them $82 \%$ said they take cheese in their diet but $44 \%$ of these females take the cheese rarely in their diet. Most of the females (86\%) never got themselves tested for vitamin D levels in their body. $80 \%$ of them are suffering with leg pain/ back pain or bone pain. Majority of the females $(60 \%)$ are not aware that vitamin D deficiency can occur at any age. 
Table No. 2: Awareness about role of Vitamin D in curing the Calcium deficiency

\begin{tabular}{|c|c|}
\hline \multicolumn{2}{|c}{$\mathrm{N}=100$} \\
\hline Level of awareness & Frequency \& percentage \\
\hline Aware & $44 \%$ \\
\hline Unaware & $56 \%$ \\
\hline
\end{tabular}

Table No. 2 shows that Most of them (56\%) are also not aware that vitamin D helps in curing the calcium deficiency.

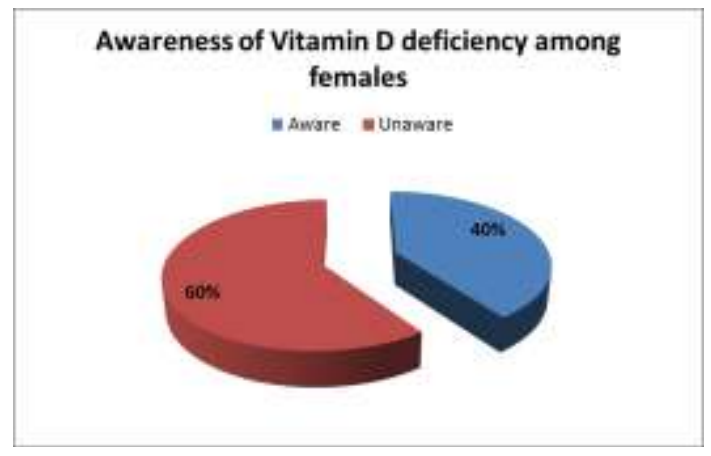

\section{Discussion}

This study revealed that majority of the study population is not aware of the vitamin D deficiency and its role in resolving the calcium deficiency. Most of the females never tested themselves for the vitamin D levels in their body. Majority of the females are suffering from the back pain/leg pain or bone pain which can be due to the deficient vitamin D levels. The dairy products consumption is also not sufficient among them.

Dr. Mona alfadeel et al conducted a study about Awareness of Vitamin D Deficiency among Female in ALMAAREFA College in 2013-2014 and found that the most of the women were aware of the vitamin D deficiency and its functions but many of them are not aware of the pregnancy related complications. This study also revealed that the although the women are aware of vitamin D deficiency but they didn't get enough vitamin $\mathrm{D}$ due to bad food habits and majority of them didn't perform the test to know their vitamin $\mathrm{D}$ levels due to the carelessness ${ }^{4}$.

Riaz Uddin et al did a study on awareness regarding the importance of calcium and vitamin D among the undergraduate pharmacy students in Bangladesh and concluded that he pharmacy students have lack of knowledge about calcium and vitamin $\mathrm{D}$ and thus it can be clearly predicted that the condition of general people may be worse ${ }^{5}$.

\section{Conclusion}

This study concludes that the study population is not aware of the vitamin D deficiency and most of the females never underwent the lab test for vitamin D levels. Majority of the females in this study are also suffering from the bone pain/leg pain or back pain. Considering the findings of the study it is of utmost importance to organize the awareness programmes to make them aware of the vitamin D deficiency, risk factors and the protective \& preventive measure for the same as the females are prone to develop the osteoporosis, osteomalacia and other systemic diseases.

\section{References}

[1] Ritu G. Ajay Gupta. Vitamin D Deficiency in India: Prevalence, Causalities and Interventions. Nutrients. 2014 Feb; 6(2): $729-775$.

[2] Petrina D'Souza. Why most Indians are vitamin D deficient. Hindustan Times, Mumbai | Updated: Apr 05, 2013 17:58 IST. Accessed on June 28, 2016.

[3] Floor Christie. Linda Mason. Knowledge, attitude and practice regarding vitamin D deficiency among female students in Saudi Arabia: A qualitative exploration. International Journal of Rheumatic Diseases. August 2011. 14(3):e22-9 .

[4] Alfadeel Mona et al, International Journal of Healthcare Sciences A Study about Awareness of Vitamin D Deficiency among Female in ALMAAREFA College in 2013-201. ISSN 2348-5728 (Online). Vol. 3, Issue 2, pp: (251-257), Month: October 2015 March 2016.

[5] Riaz Uddin. Naz Hasan Huda. YeakutyMarzanJhanker. TasbiraJesmeen. Mohammad Zafar Imam. SalehaAkter. Awareness regarding the importance of calcium and vitamin D among the undergraduate pharmacy students in Bangladesh BMC Res Notes. 2013; $6: 134$. 